Address for Correspondence: Dr. Undurti N. Das, MD, FAMS, FRSC UND Life Sciences, 2221, NW 5th St, Battle Ground, WA 98604, USA

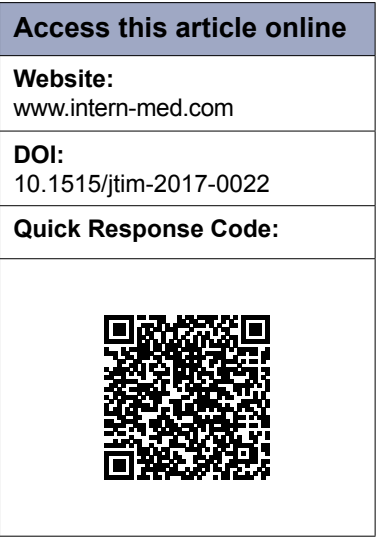

\title{
Is aortic aneurysm preventable?
}

\author{
Undurti N. Das \\ UND Life Sciences, 2221, NW 5th St, Battle Ground, WA 98604, USA
}

\section{ABSTRACT}

Abdominal aortic aneurysm (AAA) is a chronic inflammatory condition, triggered by the local accumulation of macrophages, oxidative stress and damage to the aortic wall. Pro-inflammatory eicosanoids seem to play a significant role in AAA. The pro-inflammatory events seen in AAA could be due to a deficiency of anti-inflammatory eicosanoids such as lipoxin A4 (LXA4), resolvins, protectins and maresins as a result of reduced tissue concentrations of their precursors: arachidonic acid (AA), eicosapentaenoic acid (EPA), and docosahexaenoic acid (DHA). Thus, an imbalance between pro- and anti-inflammatory eicosanoids may underlie AAA. Angiotensin-II (Ang-II), a pro-inflammatory molecule, seems to have a role in AAA. I propose that AAA is due to the local (abdominal aortic wall) deficiency of AA and other PUFAs and their anti-inflammatory metabolites especially LXA4. The beneficial action of EPA and DHA reported in the animal experimental models of AAA induced by Ang-II infusion can be attributed to their (EPA and DHA) ability to enhance the formation of not only resolvins, protectins and maresins but also LXA4. It is likely that abdominal aortic tissue (endothelial cells, smooth muscle cells and other cells) may be deficient in AA, EPA and DHA, and have defective activity of 5-, 12-, and 15-lipoxygenases and cyclooxygenase, especially COX-2 resulting in decreased formation of LXA4, resolvins, protectins and maresins. Thus, methods designed to enhance the formation of LXA4 and other anti-inflammatory eicosanoids may form a new approach to prevent and manage AAA.

Key words: aortic aneurysm, arachidonic acid, eicosapentaenoic acid, docosahexaenoic acid, lipoxin, resolvin, protectin, maresin

\section{INTRODUCTION}

In a recent experimental study performed on animals, Yoshihara et al..$^{[1]}$ showed that the dietary intake of eicosapentaenoic acid (EPA) and docosahexaenoic acid (DHA) prevented abdominal aortic aneurysm development through the inhibition of aortic and macrophage-mediated inflammation. In the model studied, the authors used angiotensin II infusion in apolipoprotein E-deficient mice to induce the development of abdominal aortic aneurysm. It is evident from their results that though both EPA and DHA could suppress the development of AAA (abdominal aortic aneurysm), in general, DHA was found to be more potent than EPA in suppressing the inflammatory markers (especially IL$1 \beta$, MCP-1, IL-6), implying that perhaps DHA is a more potent suppressor of AAA in the animal model studied. Even if one compared the changes in plasma LDL-C, HDL-C and triglycerides, the changes are more striking with DHA supplementation compared to those seen with EPA. It is known that EPA can be converted to DHA and, in turn, DHA could be retroconverted to EPA. If one compares the ratio between EPA and DHA in animals that have been supplemented with EPA and DHA, it is clear that there is a more significant increase in the plasma levels of DHA compared to EPA (in EPA supplemented animals, the ratio between EPA/DHA = 6.24, while in DHA supplemented animals the ratio between DHA/EPA is 11.73 , if EPA and DHA are equally well absorbed and the conversion of EPA to DHA and retroconversion of DHA to EPA is same/ similar). These results emphasize that perhaps, DHA is more effective than EPA in preventing the development of AAA, though the authors reported that there is no significant difference in the size of the AAA between EPA and DHA groups. Similar arguments have been presented by Meital et al. ${ }^{[2]}$ and suggested that inflammation 
plays a key role in the pathogenesis of AAA, and EPA and DHA are beneficial in the animal models of AAA in view of their anti-inflammatory actions. It was also suggested ${ }^{[2]}$ that anti-inflammatory products of EPA and DHA, such as resolvins, protectins and maresins, may have a role in this beneficial action.

\section{PUFAS AND THEIR METABOLITES, ANGIOTENSIN-II, AA AND AAA}

These results reported by Yoshihara et al. ${ }^{[1]}$ and arguments put forward by Meital et al. ${ }^{[2]}$ are interesting. Despite these impressive results and arguments, it is noteworthy that the exact mechanism(s) by which EPA and DHA can prevent AAA induced by angiotensin-II infusion remains unclear. It is not clear whether EPA and DHA themselves can bring about this beneficial action or they need to be converted to their respective anti-inflammatory compounds such as resolvins and protectins and maresins ${ }^{[3-6]}$ (resolvins E1, E2 and E3 are formed from EPA; while resolvins D1, D2, D3 and D4, protectins and maresins are formed from DHA, see Figures
1 and 2). Another important information that should have been generated is to know how much of the administered EPA and DHA were incorporated at the site of AAA and whether there is any correlation between the amount of EPA and DHA present and AAA. It will be interesting to know whether angiotensin-II decreases the content of EPA and DHA at the site of AAA and if so, whether this is reversed by the supplementation of these fatty acids. It is possible that the increased availability of EPA or DHA or both may lead to an enhanced formation of resolvins, protectins and maresins that could have reversed the development of AAA. Yet, the other possibility that needs to be considered is the action of angiotensin-II on the formation of proinflammatory prostaglandins (PGs), thromboxanes (TXs) and leukotrienes (LTs) from AA, ${ }^{[7-10]}$ an n-6 PUFA. It is likely that the supplementation of EPA and DHA displaced AA from the cell membrane phospholipid pool that can lead to an increased formation of LXA4 (see figure 3) and decreased formation of pro-inflammatory PGs, TXs and LTs (from AA). An increased presence of EPA and DHA in the cell membrane (because of their supplementation) can enhance

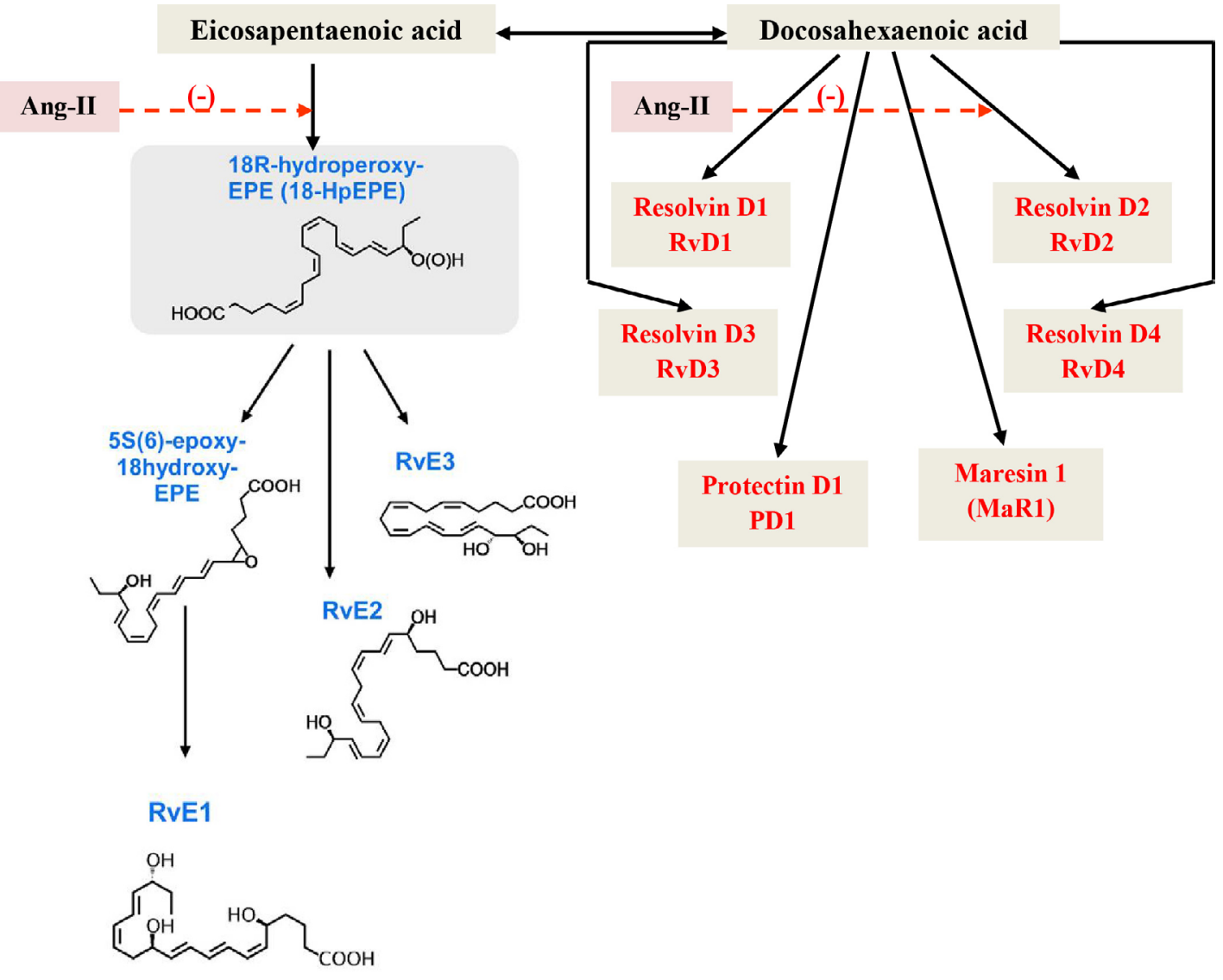

\section{E series Resolvins}

Figure 1: Scheme showing the formation of resolvins from EPA and conversion and retroconversion between EPA and DHA and formation of resolvins, protectins and maresins from DHA. Angiotensin-II may block the conversion of EPA and DHA to their respective anti-inflammatory metabolites such as resolvins, protectins and maresins and enhance the formation of pro-inflammatory prostaglandins, leukotrienes and thromboxanes from EPA and arachidonic acid (AA). 


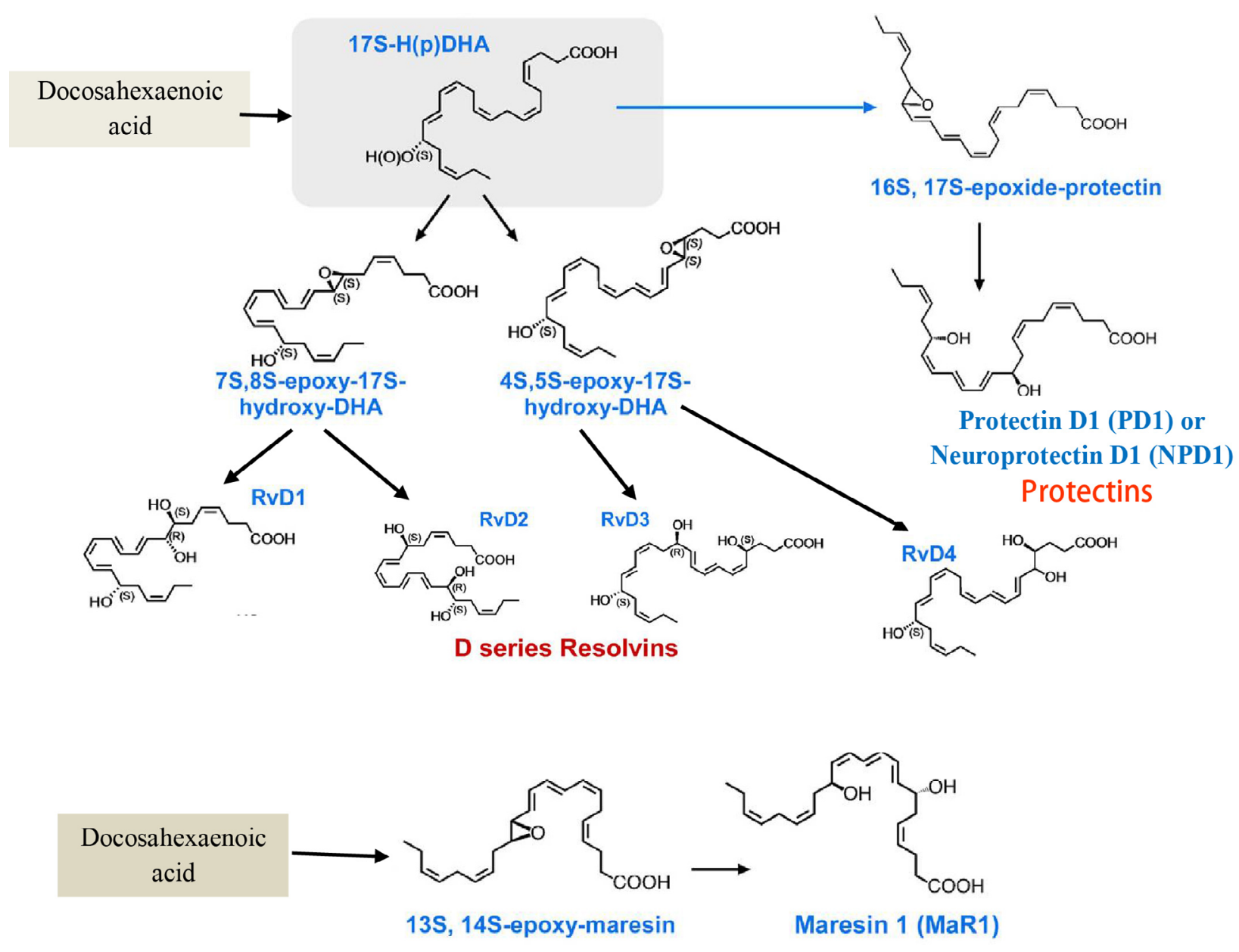

\section{Maresins}

Figure 2: Scheme showing the formation of $D$ series resolvins, protectins and maresins from DHA

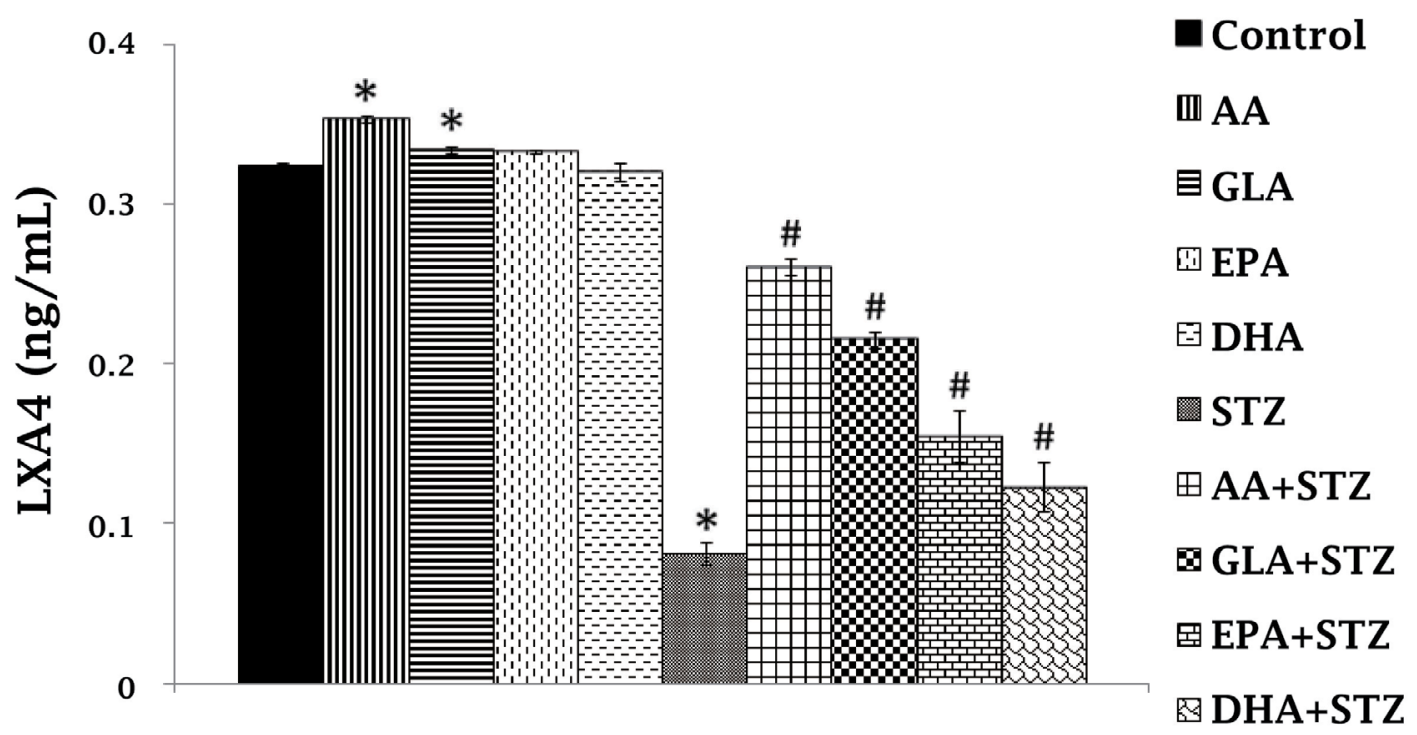

Figure 3: RIN5F (rat insulinoma) cells were treated with $10 \mu \mathrm{g} / \mathrm{mL}$ PUFAs \pm STZ $(21 \mathrm{mM}$ ) for 24 hours. The LXA4 was estimated in the supernatant of the cell cultures. All the above set of experiments were done in triplicate on two separate occasions $(n=6)$ and values are expressed as mean $\pm S E M$. ${ }^{*} P \leq 0.05$ compared to untreated control, \# P $\leq 0.05$ compared to STZ. This data is taken from Ref. 17. 
the formation of anti-inflammatory resolvins, protectins and maresins. Even though AA forms the precursor to the anti-inflammatory lipoxin A4 (LXA4), the presence of angiotensin-II, a pro-inflammatory molecule ${ }^{[11-13]}$ perhaps may drive the generation of pro-inflammatory metabolites from AA instead of anti-inflammatory LXA4. In a previous study ${ }^{[14]}$ we showed that PUFAs inhibit the activity of angiotensin converting enzyme (ACE) that is needed for the formation of Ang-II. This implies that there could occur a balance between various PUFAs and ACE activity and whenever tissue concentrations of PUFAs are low, it may result in an increased formation of Ang-II that, in turn, may trigger events that result in the development of AAA. It has not been studied, but it is possible that LXA4, resolvins, protectins and maresins may inhibit ACE activity and reduce the formation of Ang-II. If these proposals are true, then it is likely that local deficiency of AA, EPA and DHA and their anti-inflammatory LXA4, resolvins, protectins and maresins may trigger the development of AAA. The decreased formation of resolvins, protectins and maresins at the site of AAA could be attributed to the decreased activities of 5-, 12-, and 15- lipoxygenases (LOXs) and cyclooxygenase enzymes and/or their genetic polymorphisms in the target tissues that are needed for their formation. Thus, adequate activities of COX and LOX enzymes in the target tissues are essential to obtain the purported beneficial actions of the supplemented EPA and DHA.

Resolvins/protectins/maresins are not only antiinflammatory, ${ }^{[3-6]}$ but also possess anti-fibrotic actions ${ }^{[15]}$ and inhibit the proliferation of fibroblasts ${ }^{[15]}$ and thus, angiotensin-II-induced AAA could be prevented. Since angiotensin-II has pro-inflammatory actions ${ }^{[11-13]}$ and resolvins, protectins and maresins are anti-inflammatory in nature, this may explain the ability of EPA and DHA to prevent AAA. It is likely that local balance between pro- and anti-inflammatory lipid mediators (PGs, TXs, LTs $v s$. resolvins, protectins and maresins) ultimately determines the initiation and progression or prevention of AAA. Extending this argument further, it is important to estimate the plasma and aortic tissue (obtained at the time of surgery) levels of EPA and DHA and PGs, LTs, TXs and resolvins, protectins and maresins in patients with aortic aneurysms and correlate the same to the severity of the disease.

\section{ARE AA AND LXA4 CRUCIAL TO PREVENT AAA?}

In this context, it is noteworthy that there is substantial evidence to suggest that AA and LXA4 may have a more critical role in AAA compared to EPA and DHA and heir anti-inflammatory metabolites. Pillai et al. ${ }^{[16]}$ reported that in patients undergoing AAA surgery, there is a temporal relationship for local acting peptides: VEGF, IL-10 and TGF- $\beta$ and lipid mediators. Furthermore, the patients of AAA could be divided into two broad categories based on their lipid profile - one being an early resolver population and the other a delayed resolver population, though both groups eventually showed full recovery. It is of interest to note that an early resolver population showed consistently higher levels of 5-, 12-, and 15-HETE (derived from AA, 15-HETE is the precursor of LXA4), both 17-HDHA and 14-HDHA (that could lead to the formation of resolvins and maresins) and resolvins ( $\mathrm{RvE} 1$ and $\mathrm{RvE} 2)$, and their intermediate 18-HEPE-changes that indicate activation of resolution pathways. Though both early and delayed resolver groups showed slightly different temporal profile of eicosanoids, ultimately both groups recovered well. These results emphasize the fact that an early release of anti-inflammatory lipid mediators may enhance the recovery process and LXA4 seems to play a significant role in this process in addition to the active role for resolvins protectins and maresins.

In a recent study, we reported that supplementation of EPA and DHA enhances the synthesis and release of LXA4, presumably due to their ability to displace AA from cell membrane. ${ }^{[17]}$ In view of this, it is important to note that supplementation of EPA and DHA (and presumably other unsaturated fatty acids) will not only lead to the enhanced formation of resolvins, protectins and maresins but may also result in increased LXA4 generation. Hence, it is worthwhile to investigate whether a combination of $\mathrm{AA}$ and EPA and DHA is more suitable to prevent AAA in humans.

The observation by Pillai $e t$ al. ${ }^{[16]}$ that the patients of AAA belonging to the early resolver population consistently had higher levels of 5-, 12-, and 15-HETE (derived from AA, 15-HETE is the precursor of LXA4) ( $<24$ hours); While the delayed resolver population had much lower levels of LXA4 to start with but eventually showed enhanced LXA4 levels (after 48 hours), though both groups eventually showed full recovery. It is of interest to note that early resolver population showed consistently higher levels of 5-, 12-, and 15-HETE (derived from AA), both 17-HDHA and 14-HDHA (that could lead to the formation of resolvins and maresins) and resolvins (RvE1 and RvE2) and their intermediate 18-HEPE-changes that indicate an activation of resolution pathways. Though both early and delayed resolver groups showed slightly different temporal profile of eicosanoids, ultimately both groups recovered well.

These results are in support of our studies on RIN5F (rat insulinoma) cells in vitro and streptozotocin (STZ)induced type 1 and type 2 diabetes mellitus in experimental 
animals. In these studies, we noted that STZ-induced cytotoxicity to RIN5F cells is more effectively blocked by AA and LXA4 compared to EPA, DHA, resolvins and protectins. Similarly, LXA4 was found to be the most effective eicosanoid in preventing STZ-induced type 1 and type $2 \mathrm{DM}$, while resolvins and protectins were less effective (17, 18 and unpublished data). Surprisingly, AA was found to be as effective as that of LXA4 in preventing both type 1 and type 2 DM. Subsequent studies showed that AA administration restored LXA4 levels to normal that were reduce by STZ treatment. ${ }^{[17,18]}$ These results, though on a different topic emphasize the fact that AA and LXA4 are more potent anti-inflammatory compounds compared to EPA and DHA, and resolvins and protectins; under some specific conditions, they may preferentially get converted to LXA4. In view of these observations, it will be interesting to study the effect of a combination of AA, EPA and DHA in the prevention of AAA. Such a translational work could have important therapeutic implications.

\section{INTERACTION(S) AMONG ANG-II, CYTOKINES, ROS AND PUFAS AND THEIR RELEVANCE TO AAA}

In general, AAA is induced in experimental animals employing angiotensin II (Ang-II) infusion that leads to an increase in the inflammatory markers (especially IL$1 \beta$, MCP-1, IL-6), implying that AAA is an inflammatory condition. Based on this premise, it was presumed that EPA/DHA can suppress AAA since these fatty acids and their metabolites such as resolvins and protectins

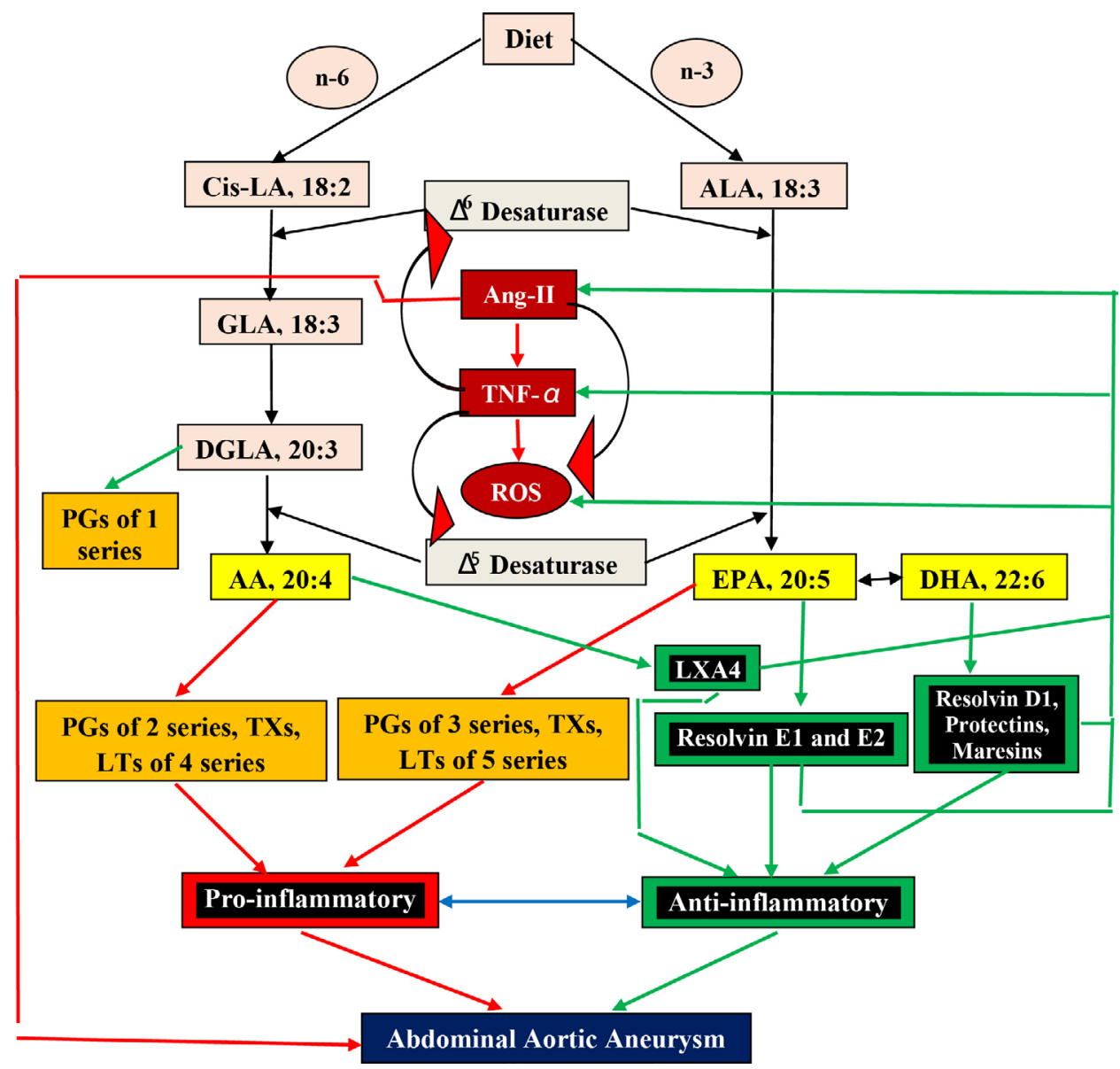

Figure 4: Scheme showing metabolism of essential fatty acids, their role in inflammation and cytoprotection of endothelial cells. Inflammation could be triggered by endogenous soluble mediators such as angiotensin-II. The activity of ACE is inhibited by various PUFAs and their anti-inflammatory metabolites such as LXA4/resolvins/ protectins/maresins. Ang-II triggers inflammation by enhancing the formation of TNF- $\alpha$ and generation of free radicals (reactive oxygen species, ROS). PUFAs and LXA4/ resolvins/protectins/maresins suppress TNF- $\alpha$ production and ROS and thus, produce their anti-inflammatory actions. TNF- $\alpha$ is known to inhibit the activities of desaturases and thus, produce EFA (essential fatty acid-LA and ALA) deficiency that may lead to decreased formation of AA, EPA and DHA and consequently reduced formation of LXA4/resolvins/protectins/maresins due to precursor deficiency. Thus, there appears to be a close interaction among Ang-II, PUFA metabolism and formation of LXA4/ resolvins/protectins/maresins, TNF- $\alpha$ and ROS generation and inflammatory process. Excess production of Ang-II, TNF- $\alpha$ and ROS may trigger inflammation and produce AAA; similarly, reduced formation of PUFAs and their anti-inflammatory metabolites LXA4/resolvins/protectins/maresins may also trigger or perpetuate inflammation and lead to the formation of AAA. Presence of adequate amounts of PUFAs/LXA4/resolvins/protectins/maresins may prevent the local inflammatory events, suppress TNF- $\alpha$ production and ROS generation and thus, preserve the integrity of aorta and prevent AAA. Similar set of events may also play a role in the pathogenesis of type 1 and type 2 diabetes mellitus, pre-eclampsia and hypertension. 
have anti-inflammatory actions. In this context, it is interesting to note that Ang-II enhances tumor necrosis factor- $\alpha$ (TNF- $\alpha$ ) production and augments free radical generation, ${ }^{[19-21]}$ events that trigger the inflammation that may explain the pro-inflammatory actions of Ang-II (Figure 4) that may underlie its (Ang-II) ability to produce AAA. In a previous study, we showed that PUFAs inhibit the activity of angiotensin-converting enzyme ${ }^{[14]}$ (ACE) and thus, reduce the formation of Ang-II and thus, suppress the inflammatory events that are associated with the renin-angiotensin-aldosterone system (RAS). In addition, PUFAs and their metabolites LXA4, resolvins, protectins and maresins are known to inhibit TNF- $\alpha$ production and suppress free radical generation. Thus, RAS and Ang-II have pro-inflammatory actions, while PUFAs and their metabolites - LXA4, resolvins, protectins and maresins have anti-inflammatory actions.

Based on the preceding discussion, it can be said that the balance between RAS and Ang-II vs. PUFAs/LXA4/ resolvins/protectins/maresins may play an important role in the pathogenesis of AAA and other conditions, in which the vascular system, salt and water balance and glucose homeostasis are critical such as hypertension, preeclampsia, type 2 diabetes mellitus, obesity and metabolic syndrome. Previously, we showed that PUFAs and their metabolites play a critical role in the pathobiology of hypertension, pre-eclampsia, type 2 diabetes mellitus and metabolic syndrome ${ }^{[14,17,18,22-34]}$. We, previously showed that AA prevents chemical-induced type 1 and type 2 diabetes, partly, due to its conversion to anti-inflammatory LXA4, ${ }^{[17,18]}$ implying that the precursor PUFAs need to be converted to their specific anti-inflammatory metabolites to produce their beneficial actions. In a similar fashion, the beneficial action of AA/EPA/DHA in the prevention of AAA will depend on their conversion to anti-inflammatory LXA4, resolvins, protectins and maresins at the site of aortic aneurysm formation. Thus, it is possible that a decrease in the activities of 5-, 12-, 15-lipoxygenases and cyclooxygenase enzymes and/or their genetic polymorphisms in the target tissues (in this case, the aortic wall) may lead to a sub-optimal formation of LXA4, resolvins, protectins and maresins in the aortic tissue, resulting in local inflammation and occurrence of AAA. If these concepts are correct, it implies that the methods to deliver AA/EPA/DHA to the site of tissue damage and their conversion to specific anti-inflammatory LXA4, resolvins, protectins and maresins is critical to derive their beneficial action(s). Hence, methods need to be developed not only to specifically deliver AA/EPA/DHA to the specific site where they are needed and/or deficient but also their conversion to respective anti-inflammatory metabolites: LXA4/resolvins/protectins/maresins to obtain their much-needed beneficial actions.

\section{Conflicts of Interest}

None declared.

\section{REFERENCES}

1. Yoshihara T, Shimada K, Fukao K, Sai E, Sato-Okabayashi Y, Matsumori $\mathrm{R}$, et al. Omega 3 polyunsaturated fatty acids suppress the development of aortic aneurysms through the inhibition of macrophagemediated inflammation. Circ J 2015; 79: 1470-8.

2. Meital LT, Sandow SL, Calder PC, Russell FD. Abdominal aortic aneurysm and omega- 3 polyunsaturated fatty acids: Mechanisms, animal models, and potential treatment. Prostaglandins Leukot Essent Fatty Acids 2017; 118: 1-9.

3. Das UN. Lipoxins, resolvins, protectins, maresins and nitrolipids and their clinical implications with specific reference to cancer: Part I. Clin Lipidol 2013; 8: 437-63.

4. Das UN. Lipoxins, resolvins, protectins, maresins and nitrolipids and their clinical implications with specific reference to diabetes mellitus and other diseases: Part II. Clin Lipidol 2013; 8: 465-80.

5. Deng B, Wang CW, Arnardottir HH, Li Y, Cheng CYC, Dalli J, et al. Maresin biosynthesis and identification of maresin 2, a new antiinflammatory and pro-resolving mediator from human macrophages. PLOS One 2014; 9: e102362.

6. Levy BD, Clish CB, Schmidt B, Gronert K, Serhan CN. Lipid mediator class switching during acute inflammation: signals in resolution. Nat Immunol 2001; 2: 612-19.

7. Gagnon DJ, Gauthier R, Regoli D. Release of prostaglandins from the rabbit perfused kidney: effects of vasoconstrictors. Br J Pharmacol 1974; 50: $533-8$

8. Miller MJ, McNeill H, Westlin WF, Carroll MA, McGiff JC. Exaggerated renal thromboxane and prostaglandin release by angiotensin II in suprarenal aortic coarctation hypertension. Pharmacol Res 1989; 21: 201-210.

9. Cruz BV, Escalante B. Renal vascular interaction of angiotensin II and prostaglandins in renovascular hypertension. J Cardiovasc Pharmacol 1999; 34: 21-7.

10. Stanke-Labesque F, Devillier P, Bedouch P, Cracowski JL, Chavanon O, Bessard G. Angiotensin II-induced contractions in human internal mammary artery: effects of cyclooxygenase and lipoxygenase inhibition. Cardiovasc Res 2000; 47: 376-83.

11. Das UN. Is angiotensin-II an endogenous pro-inflammatory molecule? Med Sci Monit 2005; 11: RA155-62.

12. Phillips MI, Kagiyama S. Angiotensin II as a pro-inflammatory mediator. Curr Opin Investig Drugs 2002; 3: 569-77.

13. Das UN. Angiotensin-II behaves as an endogenous pro-inflammatory molecule. J Assoc Physicians India 2005; 53: 472-66.

14. Vijay Kumar K, Das UN. Effect of cis-unsaturated fatty acids, prostaglandins and free radicals on angiotensin converting enzyme activity in vitro. Proc Soc Exp Biol Med 1997; 214: 374-9.

15. Qu X, Zhang X, Yao J, Song J, Nikolic-Paterson DJ, Li J. Resolvins E1 and D1 inhibit interstitial fibrosis in the obstructed kidney via inhibition of local fibroblast proliferation. J Pathol 2012; 228: 506-519.

16. Pillai PS, Leeson S, Porter TF, Owens CD, Kim JM, Conte MS, et al. Chemical mediators of inflammation and resolution in post-operative abdominal aortic aneurysm patients. Inflammation 2012; 35: 98-113.

17. Naveen KVG, Naidu VGM, Das UN. Arachidonic acid and lipoxinA4 attenuate streptozotocin-induced cytotoxicity to RIN5 F cells in vitro and type 1 and type 2 diabetes mellitus in vivo. Nutrition 2017; 35: 61-80.

18. Naveen KVG, Naidu VGM, Das UN. Arachidonic acid and lipoxin A4 attenuate alloxan-induced cytotoxicity to RIN5F cells in vitro and type 1 diabetes mellitus in vivo. BioFactors, in press.

19. Touyz RM. Reactive oxygen species as mediators of calcium signaling by angiotensin II: implications in vascular physiology and pathophysiol- 
ogy. Antioxid Redox Signal 2005; 7: 1302-14.

20. Duerrschmid C, Crawford JR, Reineke E, Taffet GE, Trial J, Entman $\mathrm{ML}$, et al. TNF receptor 1 signaling is critically involved in mediating angiotensin-II-induced cardiac fibrosis. J Mol Cell Cardiol 2013; 57: 59-67.

21. Asamizu S, Urakaze M, Kobashi C, Ishiki M, Norel Din AK, Fujisaka S, et al. Angiotensin II enhances the increase in monocyte chemoattractant protein-1 production induced by tumor necrosis factor-alpha from 3T3-L1 preadipocytes. J Endocrinol 2009; 202: 199-205.

22. Das UN. Cytokines, angiogenic and anti-angiogenic factors and bioactive lipids in pre-eclampsia. Nutrition 2015; 31: 1083-95.

23. Das UN. Minerals, trace elements and vitamins interact with essential fatty acids and prostaglandins to prevent hypertension, thrombosis, hypercholesterolemia and atherosclerosis and their attendant complications. IRCS J Med Sci 1985; 13: 684-9.

24. Prabha PS, Das UN, Koratkar R, Sagar PS, Ramesh G. Free Radicals, lipid peroxidation and essential fatty acids in uncontrolled essential hypertension. Prostaglandins Leukot Essen Fatty Acids 1990; 41: 27-33.

25. Das UN. Essential fatty acid metabolism in patients with essential hypertension, diabetes mellitus and coronary heart disease. Prostaglandins Leukot Essen Fatty Acids 1995; 52: 387-91.

26. Das UN. Nutritional factors in the pathobiology of human essential hypertension. Nutrition 2001; 17: 337-46.
27. Das UN. Long-chain polyunsaturated fatty acids interact with nitric oxide, superoxide anion, and transforming growth factor- $\beta$ to prevent human essential hypertension. Eur J Clin Nutr 2004; 58: 195-203.

28. Das UN. Metabolic syndrome X: an inflammatory condition? Curr Hypertens Rep 2004; 6: 66-73.

29. Das UN. Risk of type 2 diabetes mellitus in those with hypertension. Eur Heart J 2008; 29: 952-3.

30. Das UN. Interaction(s) of polyunsaturated fatty acids with dietary protein and its relationship to the pathogenesis of hypertension. Am J Hypertension 2010; 23: 111-2.

31. Das UN. Essential fatty acids and their metabolites in the context of hypertension. Hypertension Res 2010; 33: 782-5.

32. Das UN. Pre(peri)-natal w-3 PUFA deficiency-induced hypertension and its broader implications. Hypertension Res 2012; 35: 375-9.

33. Das UN. Renal sympathetic denervation for resistant hypertension - An alternate view. Med Hypotheses 2013; 81: 1135-6.

34. Das UN. Renin-angiotensin-aldosterone system in insulin resistance and metabolic syndrome. J Transl Int Med 2016; 4: 66-72.

How to cite this article: Das UN. Is aortic aneurysm preventable?. J Transl Intern Med 2017; 5: 72-78. 